$\begin{array}{llll}\text { I } & \mathbf{F} & \mathbf{I} & \mathbf{G}\end{array}$

RESEA R C H

R E P O R T
INSTITUT FÜR INFORMATIK

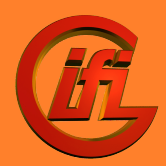

\title{
Properties of Right One-Way Jumping Finite Automata
}

\author{
Simon Beier Markus Holzer
}

IFIG RESEARCH REPORT 1802

MARCH 2018

Institut für Informatik

JLU Gießen

Arndtstraße 2

35392 Giessen, Germany

Tel: +49-641-99-32141

Fax: +49-641-99-32149

mail@informatik.uni-giessen.de

www.informatik.uni-giessen.de

\author{
JUSTUS-LIEBIG-





\title{
IFIG RESEARCH REPORT
}

IFIG ReSEARCh Report 1802, March 2018

\section{Properties of Right One-Way Jumping Finite Automata}

\author{
Simon Beier and Markus Holzer ${ }^{1}$ \\ Institut für Informatik, Universität Giessen \\ Arndtstraße 2, 35392 Giessen, Germany
}

\begin{abstract}
Right one-way jumping finite automata (ROWJFAs), were recently introduced in [H. Chigahara, S. Z. Fazekas, A. Yamamura: One-Way Jumping Finite Automata, Internat. J. Found. Comput. Sci., 27(3), 2016] and are jumping automata that process the input in a discontinuous way with the restriction that the input head reads deterministically from left-to-right starting from the leftmost letter in the input and when it reaches the end of the input word, it returns to the beginning and continues the computation. We solve most of the open problems of these devices. In particular, we characterize the family of permutation closed languages accepted by ROWJFAs in terms of Myhill-Nerode equivalence classes. Using this, we investigate closure and non-closure properties as well as inclusion relations to other language families. We also give more characterizations of languages accepted by ROWJFAs for some interesting cases.

Categories and Subject Descriptors according to ACM Computing Classification System: F.1.1 [Computation by Abstract Devices]: Models of Computation-Automata; F.4.3 [Mathematical Logic and Formal Languages]: Formal Languages-Classes Defined by Grammars or Automata; F.4.3 [Mathematical Logic and Formal Languages]: Formal LanguagesOperations on Languages
\end{abstract}

MSC Classification: 68Q45 Formal languages and automata

Additional Key Words and Phrases: Jumping Finite Automata, One-Way Restriction, Characterizations, Inclusion Relations, Closure Properties

\footnotetext{
${ }^{1}$ E-mail: \{simon.beier,markus.holzer \}@informatik.uni-giessen.de

Copyright (c) 2018 by the authors
} 


\section{Introduction}

Jumping finite automata [11] are a machine model for discontinuous information processing. Roughly speaking, a jumping finite automaton is an ordinary finite automaton, which is allowed to read letters from anywhere in the input string, not necessarily only from the left of the remaining input. In a series of papers $[1,6,7,14]$ different aspects of jumping finite automata were investigated, such as, e.g., inclusion relations, closure and non-closure results, decision problems, computational complexity of jumping finite automata problems, etc. Shortly after the introduction of jumping automata a variant of this machine model was defined, namely (right) one-way jumping finite automata [3]. There the device moves the input head deterministically from left-to-right starting from the leftmost letter in the input and when it reaches the end of the input word, it returns to the beginning and continues the computation. As in the case of ordinary jumping finite automata inclusion relations to well-known formal language families, closure and non-closure results under standard formal language operations were investigated. Nevertheless, a series of problems on right one-way jumping automata (ROWJFAs) remained open in [3]. This is the starting point of our investigation.

First we develop a characterization of (permutation closed) languages that are accepted by ROWJFAs in terms of the Myhill-Nerode relation. It is shown that the permutation closed language $L$ belongs to ROWJ, the family of all languages accepted by ROWJFAs, if and only if $L$ can be written as the finite union of Myhill-Nerode equivalence classes. Observe, that the overall number of equivalence classes can be infinite. This result nicely contrasts the characterization of regular languages, which requires that the overall number of equivalence classes is finite. The characterization allows us to identify languages that are not accepted by ROWJFAs, which are useful to prove non-closure results on standard formal language operations. In this way we solve all of the open problems from [3] on the inclusion relations of ROWJFAs languages to other language families and on their closure properties. It is shown that the family ROWJ is an anti-abstract family of languages (anti-AFL), that is, it is not closed under any of the operations $\lambda$-free homomorphism, inverse homomorphism, intersection with regular sets, union, concatenation, or Kleene star. This is a little bit surprising for a language family defined by a deterministic automaton model. Although anti-AFLs are sometimes referred to an "unfortunate family of languages" there is linguistical evidence that such language families might be of crucial importance, since in [4] it was shown that the family of natural languages is an anti-AFL. On the other hand, the family pROWJ, of all permutation closed languages in ROWJ, almost form an anti-AFL, since this language family is closed under inverse homomorphism. Moreover, we obtain further characterizations of languages accepted by ROWJFAs. For instance, we show that

1. language $w L$ is in ROWJ if and only if $L$ is in ROWJ,

2. language $L w$ is in ROWJ if and only if $L$ is regular, and

3. language $L_{1} L_{2}$ is in ROWJ if and only if $L_{1}$ is regular and $L_{2}$ is in ROWJ, where $L_{1}$ and $L_{2}$ have to fulfil some further easy pre-conditions.

The latter result is in similar vein as a result in [9] on linear context-free languages, where it was shown that $L_{1} L_{2}$ is a linear context-free language if and only if $L_{1}$ is regular and $L_{2}$ at most linear context free. Finally another characterization is given for letter bounded ROWJFA languages, namely, the language $L \subseteq a_{1}^{*} a_{2}^{*} \ldots a_{n}^{*}$ is in ROWJ if and only if $L$ is regular. This result nicely generalizes the fact that every unary language accepted by an ROWJFA is regular.

The paper is organized as follows: in the next section we introduce the necessary notations on (one-way) jumping finite automata. Then we prove a characterization of the language family ROWJ in terms of the Myhill-Nerode equivalence relation in Section 3. Then Section 4 
is devoted to inclusion relations between ROWJ and standard language families from formal language theory. There it is shown that the language family ROWJ is incomparable to the family JFA, of all languages accepted by jumping finite automata, solving an open problem from [3]. Closure properties of the language family in question and their permutation closed variant are investigated in Section 5. Finally, in Section 6 more characterizations of languages accepted by ROWJFAs are developed.

\section{Preliminaries}

We assume the reader to be familiar with the basics in automata and formal language theory as contained, for example, in [10]. Let $\mathbb{N}=\{0,1,2, \ldots\}$ be the set of non-negative integers. We use $\subseteq$ for inclusion, and $\subset$ for proper inclusion. Let $\Sigma$ be an alphabet. Then $\Sigma^{*}$ is the set of all words over $\Sigma$, including the empty word $\lambda$. For a language $L \subseteq \Sigma^{*}$ define the set $\operatorname{perm}(L)=\cup_{w \in L} \operatorname{perm}(w)$, where $\operatorname{perm}(w)=\left\{v \in \Sigma^{*} \mid v\right.$ is a permutation of $\left.w\right\}$. Then a language $L$ is called permutation closed if $L=\operatorname{perm}(L)$. The length of a word $w \in \Sigma^{*}$ is denoted by $|w|$. For the number of occurrences of a symbol $a$ in $w$ we use the notation $|w|_{a}$. We denote the powerset of a set $S$ by $2^{S}$. For $\Sigma=\left\{a_{1}, a_{2}, \ldots, a_{k}\right\}$, the Parikh-mapping $\psi: \Sigma^{*} \rightarrow \mathbb{N}^{k}$ is the function $w \mapsto\left(|w|_{a_{1}},|w|_{a_{2}}, \ldots,|w|_{a_{k}}\right)$. A language $L \subseteq \Sigma^{*}$ is called semilinear if its Parikh-image $\psi(L)$ is a semilinear subset of $\mathbb{N}^{k}$, a definition of those can be found in [8].

The elements of $\mathbb{N}^{k}$ can be partially ordered by the $\leq$-relation on vectors. For $\boldsymbol{x}, \boldsymbol{y} \in \mathbb{N}^{k}$ we write $\boldsymbol{x} \leq \boldsymbol{y}$ if all components of $\boldsymbol{x}$ are less or equal to the corresponding components of $\boldsymbol{y}$. The value $\|\boldsymbol{x}\|$ is the maximum norm of $\boldsymbol{x}$, that is, $\left\|\left(\boldsymbol{x}_{1}, \boldsymbol{x}_{2}, \ldots, \boldsymbol{x}_{k}\right)\right\|=\max \left\{\left|\boldsymbol{x}_{i}\right| \mid 1 \leq i \leq k\right\}$.

Let $\Sigma$ be an alphabet and $v, w \in \Sigma^{*}$. We say that word $v$ is a prefix of $w$ if there is an $x \in \Sigma^{*}$ with $w=v x$ and $v$ is a sub-word of $w$ if there are $x_{1}, x_{2}, \ldots, x_{n}, y_{1}, y_{2}, \ldots, y_{n+1} \in \Sigma^{*}$ with $v=x_{1} x_{2} \cdots x_{n}$ and $w=y_{1} x_{1} y_{2} x_{2} \cdots y_{n} x_{n} y_{n+1}$, for some $n \geq 0$. A language $L \subseteq \Sigma^{*}$ is called prefix-free if and only if there are no words $v, w \in L$ such that $v \neq w$ and $v$ is a prefix of $w$.

For an alphabet $\Sigma$ and a language $L \subseteq \Sigma^{*}$, let $\sim_{L}$ be the Myhill-Nerode equivalence relation on $\Sigma^{*}$. So, for $v, w \in \Sigma^{*}$, we have $v \sim_{L} w$ if and only if $v u \in L \Leftrightarrow w u \in L$, for all $u \in \Sigma^{*}$, holds. For $w \in \Sigma^{*}$, we call the equivalence class $[w]_{\sim_{L}}$ positive if and only if $w \in L$. Otherwise, the equivalence class $[w]_{\sim_{L}}$ is called negative.

A deterministic finite automaton (DFA) is defined as a tuple $A=(Q, \Sigma, R, s, F)$, where $Q$ is the finite set of states, $\Sigma$ is the finite input alphabet, $\Sigma \cap Q=\emptyset, R$ is a partial function from $Q \times \Sigma$ to $Q, s \in Q$ is the start state, and $F \subseteq Q$ is the set of final states. Elements of $R$ are referred to a rules of $A$ and we write $p y \rightarrow q \in R$ instead of $R(p, y)=q$. A configuration of $A$ is a string in $Q \Sigma^{*}$. A DFA makes a transition from configuration paw to configuration $q w$ if $p a \rightarrow q \in R$, where $p, q \in Q, a \in \Sigma$, and $w \in \Sigma^{*}$. We denote this by $p a w \vdash_{A} q w$ or just $p a w \vdash q w$ if it is clear which DFA we are referring to. In the standard manner, we extend $\vdash$ to $\vdash^{n}$, where $n \geq 0$. Let $\vdash^{+}$ and $\vdash^{*}$ denote the transitive closure of $\vdash$ and the transitive-reflexive closure of $\vdash$, respectively. Then, the language accepted by $A$ is $L(A)=\left\{w \in \Sigma^{*} \mid \exists f \in F: s w \vdash^{*} f\right\}$. We say that $A$ accepts $w \in \Sigma^{*}$ if $w \in L(A)$ and that $A$ rejects $w$ otherwise. The family of languages accepted by DFAs is referred to as REG.

A jumping finite automaton (JFA) is a tuple $A=(Q, \Sigma, R, s, F)$, where $Q, \Sigma, R, s$, and $F$ are the same as in the case of DFAs. A configuration of $A$ is a string in $\Sigma^{*} Q \Sigma^{*}$. The binary jumping relation, symbolically denoted by $\curvearrowright_{A}$, over $\Sigma^{*} Q \Sigma^{*}$ is defined as follows. Let $x, z, x^{\prime}, z^{\prime}$ be strings in $\Sigma^{*}$ such that $x z=x^{\prime} z^{\prime}$ and $p y \rightarrow q \in R$. Then, the automaton $A$ makes a jump from $x p y z$ to $x^{\prime} q z^{\prime}$, symbolically written as $x p y z \curvearrowright_{A} x^{\prime} q z^{\prime}$ or just $x p y z \curvearrowright x^{\prime} q z^{\prime}$ if it is clear which JFA we are referring to. In the standard manner, we extend $\curvearrowright$ to $\curvearrowright^{n}$, where $n \geq 0$. 
Let $\curvearrowright^{+}$and $\curvearrowright^{*}$ denote the transitive closure of $\curvearrowright$ and the transitive-reflexive closure of $\curvearrowright$, respectively. Then, the language accepted by $A$ is $L(A)=\left\{u v \mid u, v \in \Sigma^{*}, \exists f \in F: u s v \curvearrowright^{*} f\right\}$. We say that $A$ accepts $w \in \Sigma^{*}$ if $w \in L(A)$ and that $A$ rejects $w$ otherwise. Let JFA be the family of all languages that are accepted by JFAs.

A right one-way jumping finite automaton (ROWJFA) is a tuple $A=(Q, \Sigma, R, s, F)$, where the elements $Q, \Sigma, R, s$, and $F$ are defined as in a DFA. A configuration of $A$ is a string in $Q \Sigma^{*}$. The right one-way jumping relation, symbolically denoted by $\circlearrowright_{A}$, over $Q \Sigma^{*}$ is defined as follows. For $p \in Q$ we set

$$
\Sigma_{p}=\Sigma_{R, p}=\{b \in \Sigma \mid p b \rightarrow q \in R \text { for some } q \in Q\} .
$$

Now, let $p a \rightarrow q \in R, x \in\left(\Sigma \backslash \Sigma_{p}\right)^{*}$, and $y \in \Sigma^{*}$. Then, the ROWJFA $A$ makes a jump from the configuration pxay to the configuration $q y x$, symbolically written as pxay $\circlearrowright_{A} q y x$. We simply write pxay $\circlearrowright q y x$ if it is clear which ROWJFA we are referring to. In the standard manner, we extend $\circlearrowright$ to $\circlearrowright^{n}$, where $n \geq 0$. Let $\circlearrowright^{+}$and $\circlearrowright^{*}$ denote the transitive closure of $\circlearrowright$ and the transitive-reflexive closure of $\circlearrowright$, respectively. The language accepted by $A$ is the set $L(A)=\left\{w \in \Sigma^{*} \mid \exists f \in F: s w \circlearrowright^{*} f\right\}$. We say that $A$ accepts $w \in \Sigma^{*}$ if $w \in L(A)$ and that $A$ rejects $w$ otherwise. Let ROWJ be the family of all languages that are accepted by ROWJFAs. Furthermore, for $n \geq 0$, be the class of all languages accepted by ROWJFAs with at most $n$ accepting states is referred to as $\mathbf{R O W} \mathbf{J}_{\mathbf{n}}$.

Besides the above mentioned language families let $\mathbf{F I N}, \mathbf{D C F}, \mathbf{C F}$, and $\mathbf{C S}$ be the families of finite, deterministic context-free, context-free, and context-sensitive languages. Moreover, we are interested in permutation closed language families. These language families are referred to by a prefix p. E.,g., pROWJ denotes the language family of all permutation closed ROWJ languages.

Sometimes, for a DFA $A$, we will also consider the relations $\curvearrowright$ and $\circlearrowright$, that we get by interpreting $A$ as a JFA or a ROWJFA. The following three languages are associated to $A$ :

- $L_{D}(A)$ is the language accepted by $A$, interpreted as an ordinary DFA.

- $L_{J}(A)$ is the language accepted by $A$, interpreted as an JFA.

- $L_{R}(A)$ is the language accepted by $A$, interpreted as an ROWJFA.

From a result in [12] and from [3, Theorem 10], we get

$$
L_{D}(A) \subseteq L_{R}(A) \subseteq L_{J}(A)=\operatorname{perm}\left(L_{D}(A)\right) .
$$

As a consequence, we have JFA $=$ pJFA. We give an example of a DFA $A$ with the property that $L_{D}(A) \subset L_{R}(A) \subset L_{J}(A)$ :

Example 1. Let $A$ be the DFA

$$
A=\left(\left\{q_{0}, q_{1}, q_{2}, q_{3}\right\},\{a, b\}, R, q_{0},\left\{q_{3}\right\}\right),
$$

where $R$ consists of the rules $q_{0} b \rightarrow q_{1}, q_{0} a \rightarrow q_{2}, q_{2} b \rightarrow q_{3}$, and $q_{3} a \rightarrow q_{2}$. The automaton $A$ is depicted in Figure 1.

It holds $L_{D}(A)=(a b)^{+}$and

$$
L_{J}(A)=\operatorname{perm}\left((a b)^{+}\right)=\left\{\left.w \in\{a, b\}^{+}|| w\right|_{a}=|w|_{b}\right\} .
$$

Then again, it is not hard to see that $L_{R}(A)=\left\{\left.w \in a\{a, b\}^{*}|| w\right|_{a}=|w|_{b}\right\}$. Notice that this language is non-regular and not closed under permutation. 


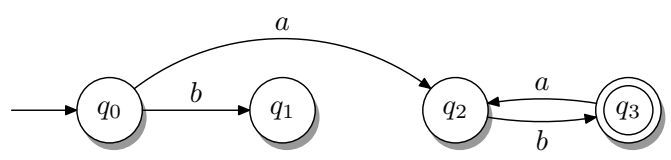

Fig. 1. The automaton $A$ with $L_{D}(A) \subset L_{R}(A) \subset L_{J}(A)$.

The following basic property will be used later on.

Lemma 2. Let $A=(Q, \Sigma, R, s, F)$ be a DFA. Consider two words $v, w \in \Sigma^{*}$, states $p, q \in Q$, and an $n \geq 0$ with $p v \circlearrowright^{n} q w$. Then, there is a word $x \in \Sigma^{*}$ such that $x w$ is a permutation of $v$, and $p x \vdash^{n} q$.

Proof. We prove this by induction on $n$. If $n=0$, we have $p v=q w$ and just set $x=\lambda$. Now, assume $n>0$ and that the lemma is true for the relation $\circlearrowright^{n-1}$. We get a state $r \in Q$, a symbol $a \in \Sigma_{r}$, and words $y \in\left(\Sigma \backslash \Sigma_{r}\right)^{*}$ and $z \in \Sigma^{*}$ such that $w=z y$ and $p v \circlearrowright^{n-1}$ ryaz $\circlearrowright q w$. By the induction hypothesis, there is a word $x^{\prime} \in \Sigma^{*}$ such that $x^{\prime} y a z$ is a permutation of $v$, and $p x^{\prime} \vdash^{n-1} r$. Set $x=x^{\prime} a$. Then, the word $x w=x^{\prime} a z y$ is a permutation of $x^{\prime} y a z$, which is a permutation of $v$. Furthermore, we get $p x=p x^{\prime} a \vdash^{n-1} r a \vdash q$. This proves the lemma.

\section{A Characterization of Permutation Closed Languages Accepted by ROWJFAs}

By the Myhill-Nerode theorem, a language $L$ is regular if and only if the Myhill-Nerode equivalence relation $\sim_{L}$ has only a finite number of equivalence classes. Moreover, the number of equivalence classes equals the number of states of the minimal DFA accepting $L$, see for example [10]. We can give a similar characterization for permutation closed languages that are accepted by an ROWJFA.

Theorem 3. Let $L$ be a permutation closed language and $n \geq 0$. Then, the language $L$ is in $\mathbf{R O W} \mathbf{J}_{\mathbf{n}}$ if and only if the Myhill-Nerode equivalence relation $\sim_{L}$ has at most $n$ positive equivalence classes.

Proof. First, assume that $L$ is in $\mathbf{R O W J}_{\mathbf{n}}$ and let $A=(Q, \Sigma, R, s, F)$ be a DFA with $|F| \leq n$ and $L_{R}(A)=L$. Consider $v, w \in L$ and $f \in F$ with $s v \circlearrowright^{*} f$ and $s w \circlearrowright^{*} f$. Lemma 2 shows that there are permutations $v^{\prime}$ and $w^{\prime}$ of $v$ and $w$ with $s v^{\prime} \vdash^{*} f$ and $s w^{\prime} \vdash^{*} f$. Because language $L$ is closed under permutation we have $v \sim_{L} v^{\prime}$ and $w \sim_{L} w^{\prime}$. Now, let $u \in \Sigma^{*}$. Thus $s v^{\prime} u \circlearrowright * f u$ and $s w^{\prime} u \circlearrowright^{*} f u$. That gives us

$$
v^{\prime} u \in L \Leftrightarrow\left(\exists g \in F: f u \circlearrowright^{*} g\right) \Leftrightarrow w^{\prime} u \in L .
$$

We have shown $v \sim_{L} v^{\prime} \sim_{L} w^{\prime} \sim_{L} w$. From $L=\bigcup_{f \in F}\left\{w \in \Sigma^{*} \mid s w \circlearrowright^{*} f\right\}$, we conclude that $\left|L / \sim_{L}\right| \leq|F| \leq n$, which means that $\sim_{L}$ has at most $n$ positive equivalence classes.

Assume now that $\sim_{L}$ has at most $n$ positive equivalence classes and let $\Sigma=\left\{a_{1}, a_{2}, \ldots, a_{k}\right\}$ be an alphabet with $L \subseteq \Sigma^{*}$. Set $L_{\lambda}=L \cup\{\lambda\}$. Define the map $S: L_{\lambda} / \sim_{L} \rightarrow 2^{\mathbb{\mathbb { N } ^ { k }}}$ through

$$
[w] \mapsto\left\{\boldsymbol{x} \in \mathbb{N}^{k} \backslash \mathbf{0} \mid \psi^{-1}(\psi(w)+\boldsymbol{x}) \subseteq L\right\} .
$$

The definition of $\sim_{L}$ and the fact that $L$ is closed under permutation make the map $S$ welldefined. Consider the relation $\leq$ on $\mathbb{N}^{k}$. For each $[w] \in L_{\lambda} / \sim_{L}$, let $M([w])$ be the set of minimal 
elements of $S([w])$. So, for every $[w] \in L_{\lambda} / \sim_{L}$ and $\boldsymbol{x} \in S([w])$, there is an $\boldsymbol{x}_{\mathbf{0}} \in M([w])$ such that $\boldsymbol{x}_{\mathbf{0}} \leq \boldsymbol{x}$. Due to [5] each subset of $\mathbb{N}^{k}$ has only a finite number of minimal elements, so the sets $M([w])$ are finite. For $i \in\{1,2, \ldots, k\}$, let $\pi_{i}: \mathbb{N}^{k} \rightarrow \mathbb{N}$ be the canonical projection on the $i$ th factor and set

$$
m_{i}=\max \left(\bigcup_{[w] \in L_{\lambda} / \sim_{L}}\left\{\pi_{i}(\boldsymbol{x}) \mid \boldsymbol{x} \in M([w])\right\}\right),
$$

where $\max (\emptyset)$ should be 0 . We have $m_{i}<\infty$, for all $i \in\{1,2, \ldots, k\}$, because of $\left|L_{\lambda} / \sim_{L}\right| \leq n+1$. Let

$$
Q=\left\{q_{[w v]_{\sim_{L}}} \mid w \in L_{\lambda}, v \in \Sigma^{*} \text { with }|v|_{a_{i}} \leq m_{i}, \text { for all } i \in\{1,2, \ldots, k\}\right\}
$$

be a set of states. The finiteness of $L_{\lambda} / \sim_{L}$ implies that $Q$ is also finite. Set

$$
F=\left\{q_{[w]_{\sim_{L}}} \mid w \in L\right\} \subseteq Q .
$$

We get $|F|=\left|L / \sim_{L}\right| \leq n$. Define the partial mapping $R: Q \times \Sigma \rightarrow Q$ by $R\left(q_{[y]_{\sim_{L}}}, a\right)=q_{[y a]_{\sim_{L}}}$, if $q_{[y a]_{\sim_{L}}} \in Q$, and $R\left(q_{[y]_{\sim_{L}}}, a\right)$ be undefined otherwise, for $a \in \Sigma$ and $y \in \Sigma^{*}$ with $q_{[y]_{\sim_{L}}} \in Q$. Consider the DFA $A=\left(Q, \Sigma, R, q_{[\lambda] \sim_{L}}, F\right)$. We will show that $L_{R}(A)=L$.

First, let $y \in L_{R}(A)$. Then, there exists $w \in L$ with $q_{[\lambda]_{\sim_{L}}} y \circlearrowright^{*} q_{[w]_{\sim_{L}}}$. From Lemma 2 it follows that there is a permutation $y^{\prime}$ of $y$ with $q_{[\lambda]_{\sim_{L}}} y^{\prime} \vdash^{*} q_{[w]_{\sim_{L}}}$. Now, the definition of $R$ tells us $y^{\prime} \sim_{L} w$. We get $y^{\prime} \in L$ and also $y \in L$, because $L$ is closed under permutation. That shows the inclusion $L_{R}(A) \subseteq L$.

Now, let $y \in \Sigma^{*} \backslash L_{R}(A)$. There are two possibilities:

1. There is $w \in \Sigma^{*} \backslash L$ with $q_{[w]_{\sim_{L}}} \in Q$ such that $q_{[\lambda]_{\sim_{L}}} y \circlearrowright^{*} q_{[w]_{\sim_{L}}}$. Then, there is a permutation $y^{\prime}$ of $y$ with $q_{[\lambda]_{\sim_{L}}} y^{\prime} \vdash^{*} q_{[w]_{\sim_{L}}}$. We get $y^{\prime} \sim_{L} w$. It follows $y^{\prime} \notin L$, which gives us $y \notin L$.

2. There are a $w \in L_{\lambda}$, a $v \in \Sigma^{*}$ with $|v|_{a_{i}} \leq m_{i}$, for all $i \in\{1,2, \ldots, k\}$, and a word $z$ with $z \in\left(\Sigma \backslash \Sigma_{q_{\left[w v \sim_{L}\right.}}\right)^{+}$such that $q_{[\lambda]_{\sim_{L}}} y \circlearrowright^{*} q_{[w v]_{\sim_{L}}} z$. By Lemma 2 there is a $y^{\prime} \in \Sigma^{*}$ such that $y^{\prime} z$ is a permutation of $y$ and satisfies $q_{[\lambda]_{\sim_{L}}} y^{\prime} \vdash^{*} q_{[w v]_{\sim_{L}}}$. We get $y^{\prime} \sim_{L} w v$. Set

$$
U=\bigcup_{t \in \Sigma^{*}}\left\{u \in \Sigma^{*} \mid u t \in \operatorname{perm}(v) \text { and } w u \in L_{\lambda}\right\}
$$

We have $\lambda \in U$. Let $u_{0} \in U$ such that $\left|u_{0}\right|=\max (\{|u| \mid u \in U\})$ and let $t_{0} \in \Sigma^{*}$ such that $u_{0} t_{0} \in \operatorname{perm}(v)$. It follows that $\left|t_{0}\right|_{a_{i}} \leq|v|_{a_{i}} \leq m_{i}$, for all $i \in\{1,2, \ldots, k\}$, and that there exists no $\boldsymbol{x} \in M\left(\left[w u_{0}\right]_{\sim_{L}}\right)$ with $\boldsymbol{x} \leq \psi\left(t_{0}\right)$. Otherwise, we would have an $x^{\prime} \in \psi^{-1}(\boldsymbol{x})$ which is a non-empty sub-word of $t_{0}$ such that $w u_{0} x^{\prime} \in L$, which implies $u_{0} x^{\prime} \in U$. However, this is a contradiction to the maximality of $\left|u_{0}\right|$. That shows that there is no $\boldsymbol{x} \in M\left(\left[w u_{0}\right]_{\sim_{L}}\right)$ with $\boldsymbol{x} \leq \psi\left(t_{0}\right)$. Let now $\boldsymbol{x}_{\mathbf{0}} \in M\left(\left[w u_{0}\right]_{\sim_{L}}\right)$. Then $\left|t_{0}\right|_{a_{j}}<\pi_{j}\left(\boldsymbol{x}_{\mathbf{0}}\right) \leq m_{j}$, for some element $j \in\{1,2, \ldots, k\}$. Because of $\left|t_{0}\right|_{a_{i}} \leq m_{i}$, for all $i$ with $i \in\{1,2, \ldots, k\}$, and since we have the equality $z \in\left(\Sigma \backslash \Sigma_{q_{[w v] \sim_{L}}}\right)^{+}=\left(\Sigma \backslash \Sigma_{q_{\left[w u_{0} t_{0}\right] \sim_{L}}}\right)^{+}$, we get $|z|_{a_{j}}=0$. That gives $\left|t_{0} z\right|_{a_{j}}<\pi_{j}\left(\boldsymbol{x}_{\mathbf{0}}\right)$ and that $\psi\left(t_{0} z\right) \geq \boldsymbol{x}_{\mathbf{0}}$ is false. So, we have shown $\psi\left(t_{0} z\right) \notin S\left(\left[w u_{0}\right]_{\sim_{L}}\right)$, which immediatley implies $w u_{0} t_{0} z \notin L$. From $w u_{0} t_{0} z \sim_{L} w v z \sim_{L} y^{\prime} z \sim_{L} y$, it follows that $y \notin L$.

We have seen $L_{R}(A)=L$. This shows that $L$ is in $\mathbf{R O W J}_{\mathbf{n}}$. 
The previous theorem allows us to determine for a lot of interesting languages whether they belong to ROWJ or not.

Corollary 4. Let $L$ be a permutation closed language. Then, the language $L$ is in ROWJ if and only if the Myhill-Nerode equivalence relation $\sim_{L}$ has only a finite number of positive equivalence classes.

An application of the last corollary is the following.

Lemma 5. The language $L=\left\{\left.w \in\{a, b\}^{*}|| w\right|_{b}=0 \vee|w|_{b}=|w|_{a}\right\}$ is not included in ROWJ.

Proof. Obviously, the language $L$ is closed under permutation. For $\sim_{L}$, the positive equivalence classes $\left[a^{0}\right],\left[a^{1}\right], \ldots$ are pairwise different, since $a^{n} b^{m} \in L$ if and only if $m \in\{0, n\}$. Corollary 4 tells us that $L$ is not in ROWJ.

There are counterexamples for both implications of Corollary 4, if we do not assume that the language $L$ is closed under permutation. For instance, set $L=\left\{a^{n} b^{n} \mid n \geq 0\right\}$, which was shown to be not in ROWJ in [3]. Then, the positive equivalence classes of $\sim_{L}$ are $[\lambda]$ and $[a b]$. On the other hand, we have:

Lemma 6. There is a language $L$ in ROWJ such that $\sim_{L}$ has an infinite number of positive equivalence classes.

Proof. Let $A$ be the ROWJFA

$$
\left(\left\{q_{0}, q_{1}, q_{2}, q_{3}, q_{4}\right\},\{a, b\}, R, q_{0},\left\{q_{2}, q_{3}\right\}\right),
$$

where $R$ consists of the rules $q_{0} b \rightarrow q_{1}, q_{1} a \rightarrow q_{2}, q_{2} a \rightarrow q_{2}, q_{1} b \rightarrow q_{3}, q_{3} a \rightarrow q_{4}$, and $q_{4} b \rightarrow q_{3}$. The ROWJFA $A$ is depicted in Figure 2. Let $n>0$. Then, we have $q_{0} a^{n} b \circlearrowright q_{1} a^{n} \circlearrowright^{+} q_{2}$, which

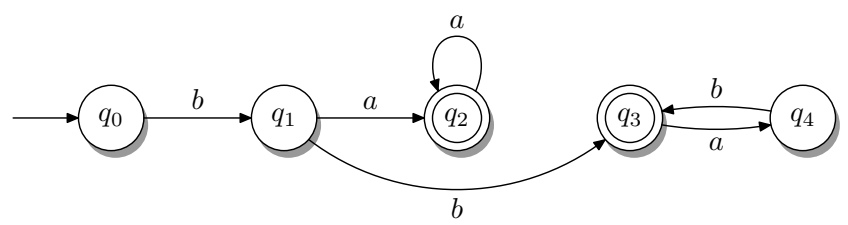

Fig. 2. The ROWJFA $A$ accepting a language that has an infinite number of positive equivalence classes w.r.t. $\sim_{L(A)}$.

gives $a^{n} b \in L(A)$. We also have

$$
q_{0} a^{n} b b b^{n} \circlearrowright q_{1} b b^{n} a^{n} \circlearrowright q_{3} b^{n} a^{n} \circlearrowright^{2} q_{3} b^{n-1} a^{n-1} \circlearrowright^{2} \cdots \circlearrowright^{2} q_{3} b^{0} a^{0} .
$$

It follows $a^{n} b b b^{n} \in L(A)$. Whenever $A$ is in state $q_{3}$, the number of read $b$ 's equals the number of read $a$ 's plus 2. That implies $a^{n} b b b^{m} \notin L(A)$, for all $m \geq 0$ with $m \neq n$. So, the positive equivalence classes $\left[a^{1} b\right]_{\sim_{L(A)}},\left[a^{2} b\right]_{\sim_{L(A)}}, \ldots$ are pairwise different. This proves the lemma.

From Corollary 4 we conclude the following equivalence.

Corollary 7. Let $L$ be a permutation closed ROWJ language over the alphabet $\Sigma$. Then, the language $L$ is regular if and only if $\Sigma^{*} \backslash L$ is in $\mathbf{R O W J}$. 
Proof. By Corollary 4, the Myhill-Nerode equivalence relation $\sim_{L}$ has only a finite number of positive equivalence classes. So, $L$ is regular if and only if $\sim_{L}$ has only a finite number of negative equivalence classes, by the Myhill-Nerode theorem. The latter condition holds if and only if $\sim_{\Sigma^{*} \backslash L}$ has only a finite number of positive equivalence classes. Again by Corollary 4 , this is equivalent to the condition that $\Sigma^{*} \backslash L$ is in $\mathbf{R O W J}$, because the complement of a permutation closed language is also permutation closed.

The previous corollary gives us:

Lemma 8. The language $\left\{\left.w \in\{a, b\}^{*}|| w\right|_{a} \neq|w|_{b}\right\}$ is not in ROWJ.

Proof. Consider the permutation closed non-regular language $L=\left\{\left.w \in\{a, b\}^{*}|| w\right|_{a}=|w|_{b}\right\}$ over the alpbabet $\Sigma=\{a, b\}$. In [3], it was shown that $L$ is in ROWJ. Now, by Corollary 7 , the language

$$
\Sigma^{*} \backslash L=\left\{\left.w \in\{a, b\}^{*}|| w\right|_{a} \neq|w|_{b}\right\}
$$

is not in ROWJ.

Having the statement of Theorem 3, it is natural to ask, which numbers arise as the number of positive equivalence classes of the Myhill-Nerode equivalence relation $\sim_{L}$ of a permutation closed language $L$. The answer is, that all natural numbers arise this way, even if we restrict ourselves to some special families:

Theorem 9. For each $n>0$, there is a permutation closed language which is (1) finite, (2) regular, but infinite, (3) context-free, but non-regular, (4) non-context-free such that the corresponding Myhill-Nerode equivalence relation has exactly $n$ positive equivalence classes.

Proof. For each $n>0$, set

$$
\begin{aligned}
L_{n} & =\left\{a^{m} \mid m<n\right\}, \\
M_{n} & =\left\{a^{m} \mid m \bmod (n+1) \neq n\right\}, \\
N_{n} & =\left\{\left.w \in\{a, b\}^{+}|| w\right|_{a}=|w|_{b}\right\} \cup\left\{c^{m} \mid 0<m<n\right\}, \\
O_{n} & =\left\{\left.w \in\{a, b, c\}^{+}|| w\right|_{a}=|w|_{b}=|w|_{c}\right\} \cup\left\{d^{m} \mid 0<m<n\right\} .
\end{aligned}
$$

All these languages are closed under permutation. Obviously, the language $L_{n}$ is finite. The positive equivalence classes of $\sim_{L_{n}}$ are $\left[a^{0}\right],\left[a^{1}\right], \ldots,\left[a^{n-1}\right]$.

While $M_{n}$ is infinite, the equivalence classes of $\sim_{M_{n}}$ are $\left[a^{0}\right],\left[a^{1}\right], \ldots,\left[a^{n}\right]$. So, the language $M_{n}$ is regular, by the Myhill-Nerode Theorem. Only the last mentioned equivalence class is negative. Therefore, there are exactly $n$ positive equivalence classes of $\sim_{M_{n}}$.

For $\sim_{N_{n}}$, the equivalence classes $\left[a^{0}\right],\left[a^{1}\right], \ldots$ are pairwise different. So, the language $N_{n}$ is non-regular, by the Myhill-Nerode Theorem. It is context-free, because it is the union of a well known context-free language and a finite language. The positive equivalence classes of the relation $\sim_{N_{n}}$ are $[a b],\left[c^{1}\right],\left[c^{2}\right], \ldots,\left[c^{n-1}\right]$.

If $O_{n}$ was context-free, then

$$
O_{n} \cap\{a, b, c\}^{*}=\left\{\left.w \in\{a, b, c\}^{+}|| w\right|_{a}=|w|_{b}=|w|_{c}\right\}
$$

would also be context-free, as the intersection of a context-free and a regular language. However, the language $\left\{\left.w \in\{a, b, c\}^{+}|| w\right|_{a}=|w|_{b}=|w|_{c}\right\}$ is a well known non-context-free language. It follows, that $O_{n}$ is also non-context-free. The positive equivalence classes of the relation $\sim_{O_{n}}$ are $[a b c],\left[d^{1}\right],\left[d^{2}\right], \ldots,\left[d^{n-1}\right]$. This proves the theorem. 
The previous theorem, together with Theorem 3, implies that the language families $\mathbf{R O W} \mathbf{J}_{\mathbf{n}}$ form a proper hierarchy, even if we only consider languages out of special language families:

Corollary 10. For all $n \geq 0$, we have

$$
\begin{aligned}
\mathbf{R O W J}_{\mathbf{n}} & \subset \mathbf{R O W J}_{\mathbf{n}+\mathbf{1}}, \\
\mathbf{R O W J}_{\mathbf{n}} \cap \mathbf{F I N} & \subset \mathbf{R O W J}_{\mathbf{n}+\mathbf{1}} \cap \mathbf{F I N}, \\
\mathbf{R O W J}_{\mathbf{n}} \cap(\mathbf{R E G} \backslash \mathbf{F I N}) & \subset \mathbf{R O W J}_{\mathbf{n}+\mathbf{1}} \cap(\mathbf{R E G} \backslash \mathbf{F I N}), \\
\mathbf{R O W J}_{\mathbf{n}} \cap(\mathbf{C F} \backslash \mathbf{R E G}) & \subset \mathbf{R O W J}_{\mathbf{n}+\mathbf{1}} \cap(\mathbf{C F} \backslash \mathbf{R E G}), \\
\mathbf{R O W J}_{\mathbf{n}} \cap(\mathbf{C S} \backslash \mathbf{C F}) & \subset \mathbf{R O W J}_{\mathbf{n}+\mathbf{1}} \cap(\mathbf{C S} \backslash \mathbf{C F}) .
\end{aligned}
$$

The statement remains valid if restricted to permutation closed languages.

\section{Inclusion Relations Between Language Families}

We investigate inclusion relations between ROWJ and other important languages families. The following inclusion relations were given in [3]:

- REG $\subset$ ROWJ,

- ROWJ and CF are incomparable,

- ROWJ $\nsubseteq$ JFA.

It was stated as an open problem if JFA $\subset$ ROWJ. We can answer this:

Theorem 11. The language families ROWJ and JFA are incomparable.

Proof. The language $\left\{\left.w \in\{a, b\}^{*}|| w\right|_{b}=0 \vee|w|_{b}=|w|_{a}\right\}$ is not included in the family ROWJ, by Lemma 5, but it belongs to JFA, because it is the permutation closure of the regular language $a^{*} \cup(a b)^{*}$. So, we get JFA $\nsubseteq \mathbf{R O W J}$. Together with the result ROWJ $\nsubseteq$ JFA from $[3]$ the incomparability of the language families ROWJ and JFA follows.

For the complexity of ROWJ, we get:

Theorem 12. The language family ROWJ is included in $\operatorname{DTIME}\left(n^{2}\right)$ and $\operatorname{DSPACE}(n)$.

Proof. Right revolving automata were described in [2]. It was shown that every language accepted by a deterministic right revolving automaton belongs to bothclasses $\operatorname{DTIME}\left(n^{2}\right)$ and $\operatorname{DSPACE}(n)$. In [3] it was proven that ROWJ is properly included in the family of languages accepted by deterministic right revolving automata.

This implies that ROWJ is properly included in CS:

Theorem 13. We have ROWJ $\subset$ CS.

Proof. From Theorem 12 we get ROWJ $\subseteq$ CS. On the other hand, ROWJ and CF are incomparable, which proves the theorem.

We also get a result for the inclusion relation between ROWJ and the family of deterministic context-free languages:

Theorem 14. The language families ROWJ and DCF are incomparable.

Proof. The families ROWJ and $\mathbf{C F}$ are incomparable, so there are non context-free languages in ROWJ. Morever, it was shown in [3] that the deterministic context-free language

$$
\left\{a^{n} b^{n} \mid n \geq 0\right\}
$$

is not accepted by any ROWJFA. 
By the famous result in [13], every context-free language is semilinear. In [3] it was proven that every language in JFA is also semilinear. This holds for ROWJ, too:

Theorem 15. Every language in ROWJ is semilinear.

Proof. For every language $L$ in ROWJ, there exists a DFA $A$ such that $L=L_{R}(A)$. From (1) we get

$$
\psi\left(L_{D}(A)\right) \subseteq \psi\left(L_{R}(A)\right) \subseteq \psi\left(L_{J}(A)\right) .
$$

Because of $L_{J}(A)=\operatorname{perm}\left(L_{D}(A)\right)$, we have $\psi\left(L_{J}(A)\right)=\psi\left(L_{D}(A)\right)$. So,

$$
\psi(L)=\psi\left(L_{R}(A)\right)=\psi\left(L_{D}(A)\right),
$$

which is a semilinear set, because $L_{D}(A)$ is regular.

We now consider inclusion relations between families of permutation closed languages. It holds

$$
\mathrm{pFIN} \subset \mathbf{p R E G} \subset \mathbf{p D C F} \subseteq \mathbf{p C F} \subset \mathbf{p C S},
$$

witness languages are $a^{*},\left\{\left.w \in\{a, b\}^{*}|| w\right|_{a}=|w|_{b}\right\}$, and $\left\{a^{2^{n}} \mid n \geq 0\right\}$. There is also a language that distinguishes $\mathbf{p D C F}$ and $\mathbf{p C F}$ :

Theorem 16. We have $\mathbf{p D C F} \subset \mathbf{p C F}$.

Proof. Consider the permutation closed language

$$
L=\left\{\left.w \in\{a, b, c\}^{*}|| w\right|_{a}=|w|_{b} \vee|w|_{b}=|w|_{c}\right\} .
$$

It is context-free as the union of two context-free languages. If $L$ was deterministic context-free, then

$$
L^{\prime}=L \cap a^{*} b^{*} c^{*}=\left\{a^{i} b^{j} c^{k} \mid(i, j, k \geq 0) \wedge(i=j \vee j=k)\right\}
$$

was also deterministic context-free as the intersection of a deterministic context-free and a regular language. However, language $L^{\prime}$ is not deterministic context-free, as shown in [10]. Hence language $L$ is also not deterministic context-free, which proves the theorem.

The next theorem places JFA in the hierarchy (2).

Theorem 17. We have pCF $\subset$ JFA $\subset$ pCS.

Proof. The first strict inclusion is seen as follows: it was shown that every context-free language is semilinear in [13], while in [3] it was proven that JFA is the family of all permutation closed semilinear languages. So, we get $\mathbf{p C F} \subseteq \mathbf{J F A}$. On the other hand, the non-context free language

$$
\left\{\left.w \in\{a, b, c\}^{*}|| w\right|_{a}=|w|_{b}=|w|_{c}\right\}
$$

is in JFA, which was shown in [11]. This proves the first inclusion.

For the second strict inclusion we argue as follows: in [11] it was proven that JFA $\subset$ CS and that all languages in JFA are closed under permutation. This gives us JFA $\subseteq$ pCS. The permutation closed context-sensitive language $\left\{a^{2^{n}} \mid n \geq 0\right\}$ is not in JFA, because it is not semilinear. 
So, we get

$$
\mathbf{p F I N} \subset \mathbf{p R E G} \subset \mathbf{p D C F} \subset \mathbf{p C F} \subset \mathbf{J F A} \subset \mathbf{p C S} .
$$

We investigate the inclusion relations of $\mathbf{p R O W J}$, now.

Theorem 18. We have $\mathbf{p R E G} \subset \mathbf{p R O W J} \subset \mathbf{J F A}$.

Proof. Since REG $\subset$ ROWJ, we have pREG $\subseteq$ pROWJ. The permutation closed, nonregular language $\left\{\left.w \in\{a, b\}^{*}|| w\right|_{a}=|w|_{b}\right\}$ was shown to be included in ROWJ in [3].

Theorem 15 implies pROWJ $\subseteq$ JFA, because JFA is the family of all permutation closed semilinear languages. On the other hand, Theorem 11 tells us that there is a language in JFA, which is not in pROWJ.

Next, we consider the inclusion relations between $\mathbf{p R O W J}$ and the language families $\mathbf{p D C F}$ and $\mathbf{p C F}$.

Theorem 19. The language family $\mathbf{p R O W J}$ is incomparable to $\mathbf{p D C F}$ and to $\mathbf{p C F}$.

Proof. From [3] we know that the permutation closed, non-context-free language

$$
\left\{\left.w \in\{a, b, c\}^{*}|| w\right|_{a}=|w|_{b}=|w|_{c}\right\}
$$

is in ROWJ. The language $L=\left\{\left.w \in\{a, b\}^{*}|| w\right|_{a}=|w|_{b}\right\}$ is in pDCF and so is $\{a, b\}^{*} \backslash L$, because pDCF is closed under complementation. Lemma 8 gives us that $\{a, b\}^{*} \backslash L$ is not in ROWJ, which proves the theorem.

Finally, we get from Theorems 11 and 18:

Theorem 20. We have pROWJ $\subset$ ROWJ .

\section{Closure Properties of ROWJ and pROWJ}

We consider closure properties of the language families ROWJ and pROWJ. Our results are summarized in Table 1.

The language family ROWJ is not closed under the operations of intersection, intersection with regular languages, reversal, concatenation, concatenation with regular languages from the right, Kleene star, Kleene plus, and substitution. All these properties were proven in [3]. In the following we will show that ROWJ is also not closed under the operations of union, union with regular languages, complement, concatenation with regular languages from the left, homomorphism, $\lambda$-free homomorphism, inverse homomorphism and permutation closure. However, we will prove one positive closure result: the family ROWJ is closed under concatenation with prefix-free regular languages from the left.

Theorem 21. The family ROWJ is not closed under union and under union with regular languages.

Proof. Consider $L_{1}=a^{*}$ and $L_{2}=\left\{\left.w \in\{a, b\}^{*}|| w\right|_{a}=|w|_{b}\right\}$. The language $L_{1}$ is in ROWJ, because it is regular, while $L_{2}$ was shown to be in ROWJ in [3]. In Lemma 5 it was shown that the union $L_{1} \cup L_{2}$ is not in ROWJ.

Next we consider the complementation operation.

Theorem 22. The family ROWJ is not closed under complement.

Proof. While $\left\{\left.w \in\{a, b\}^{*}|| w\right|_{a}=|w|_{b}\right\}$ is in ROWJ, its complement is not, which was shown in Lemma 8. 


\begin{tabular}{|l||c|c|c|c|}
\hline \hline \multirow{2}{*}{\multicolumn{1}{|c||}{ Closed under }} & \multicolumn{4}{|c|}{ Language family } \\
\cline { 2 - 5 } & REG & pROWJ & ROWJ & JFA \\
\hline \hline Union & yes & no & no & yes \\
Union with reg. lang. & yes & no & no & no \\
Intersection & yes & yes & no & yes \\
Intersection with reg. lang. & yes & no & no & no \\
Complementation & yes & no & no & yes \\
Reversal & yes & yes & no & yes \\
Concatenation & yes & no & no & no \\
Right conc. with reg. lang. & yes & no & no & no \\
Left conc. with reg. lang. & yes & no & no & no \\
Left conc. with prefix-free reg. lang. & yes & no & yes & no \\
Kleene star & yes & no & no & no \\
Kleene plus & yes & no & no & no \\
Homomorphism & yes & no & no & no \\
$\lambda$-free homomorphism & yes & no & no & no \\
Inv. homomorphism & yes & yes & no & yes \\
Substitution & yes & no & no & no \\
Permutation & no & yes & no & yes \\
\hline \hline
\end{tabular}

Table 1. Closure properties of ROWJ and pROWJ. The gray shaded results are proven in this paper. The non-shaded closure properties for REG are folklore. For ROWJ the closure/non-closure results can be found in [3] and that for the language family JFA in $[1,6,7,12]$.

From [3] we know that ROWJ is not closed under concatenation, not even under concatenation with regular languages from the right. Also, under concatenation with regular languages from the left, the family ROWJ is not closed:

Theorem 23. The family ROWJ is not closed under concatenation with regular languages from the left.

Proof. Consider the regular language $L_{1}=a^{*}$ and the ROWJ language

$$
L_{2}=\left\{\left.w \in\{a, b\}^{*}|| w\right|_{a}=|w|_{b}\right\} .
$$

Assume that there is a DFA $A=(Q,\{a, b\}, R, s, F)$ with $L_{R}(A)=L_{1} L_{2}$. For each $n \geq 0$, there is exactly one $q_{n} \in F$ with $s a^{n} \vdash^{*} q_{n}$. Because of $|F| \leq \infty$, there are $0 \leq n<m$ with $q_{n}=q_{m}$. Since the word $\lambda a^{m} b^{m}$ belongs to $L_{1} L_{2}$, there exists $q \in F$ with $s a^{m} b^{m} \vdash^{*} q_{m} b^{m} \vdash^{*} q$. This implies that $s a^{n} b^{m} \vdash^{*} q_{m} b^{m} \vdash^{*} q$, which gives us $a^{n} b^{m} \in L_{1} L_{2}$. That is a contradiction, because of $m>n$. Thus, the language $L_{1} L_{2}$ is not in ROWJ.

If we add the condition that the regular language has to be prefix-free, we get a positive closure result:

Theorem 24. The family ROWJ is closed under concatenation with prefix-free regular languages from the left.

Proof. For an alphabet $\Sigma$, let $L_{1} \subseteq \Sigma^{*}$ be a prefix-free regular language and moreover $L_{2} \subseteq \Sigma^{*}$ be a ROWJ language. If $\lambda \in L_{1}$, we have $L_{1}=\{\lambda\}$ and therefore $L_{1} L_{2}=L_{2}$. Thus, assume form now on that $\lambda \notin L_{1}$. Let $A_{1}=\left(Q_{1}, \Sigma, R_{1}, s_{1}, F_{1}\right)$ be a DFA with total transition function $R_{1}$ 
and $L_{D}\left(A_{1}\right)=L_{1}$. Moreover, let $A_{2}=\left(Q_{2}, \Sigma, R_{2}, s_{2}, F_{2}\right)$ be a DFA with $L_{R}\left(A_{2}\right)=L_{2}$ and assume $Q_{1} \cap Q_{2}=\emptyset$ without loss of generality. Consider the DFA

$$
B=\left(\left(Q_{1} \backslash F_{1}\right) \cup Q_{2}, \Sigma, S, s_{1}, F_{2}\right),
$$

where $S$ is defined as follows: for $(q, a) \in\left(Q_{1} \backslash F_{1}\right) \times \Sigma$, let $S(q, a)=R_{1}(q, a)$, if $R_{1}(q, a) \notin F_{1}$, and $S(q, a)=s_{2}$, otherwise. For $(q, a) \in Q_{2} \times \Sigma$, the value $S(q, a)$ is defined if and only if $R_{2}(q, a)$ is defined. In this case we have $S(q, a)=R_{2}(q, a)$. We will show that $L_{R}(B)=L_{1} L_{2}$.

First, let $v \in L_{1}$ and $w \in L_{2}$. So, there is a symbol $a \in \Sigma$ and states $p \in Q_{1}, q \in F_{1}$, and $r \in F_{2}$ such that $s_{1} v \vdash_{A_{1}}^{*} p a \vdash_{A_{1}} q$ and $s_{2} w \circlearrowright_{A_{2}}^{*} r$. Because $L_{1}$ is prefix-free, there are no word $x \in \Sigma^{+}$and $q^{\prime} \in F_{1}$ such that $s_{1} v \vdash_{A_{1}}^{*} q^{\prime} x$. This gives us $s_{1} v w \vdash_{B}^{*}$ paw $\vdash_{B} s_{2} w \circlearrowright_{B}^{*} r$, which implies $v w \in L_{R}(B)$.

Let now $v \in L_{R}(B)$. Since $R_{1}$ is a total function, there are a symbol $a \in \Sigma$, words $w, x \in \Sigma^{*}$, and states $p \in Q_{1} \backslash F_{1}$ and $q \in F_{2}$ such that $v=$ wax and $s_{1}$ wax $\vdash_{B}^{*} p_{a x} \vdash_{B} s_{2} x \circlearrowright_{B}^{*} q$. So, there is an $r \in F_{1}$ with $s_{1} w a \vdash_{A_{1}}^{*} p a \vdash_{A_{1}} \quad r$ and $s_{2} x \circlearrowright_{A_{2}}^{*} q$. This gives us $w a \in L_{1}$ and $x \in L_{2}$, which proves the theorem.

The previous theorem allows us for a large family of languages to show that they belong to ROWJ. From Corollary 4 and Theorem 24 it follows that:

Corollary 25. Let $\Sigma$ be an alphabet and $w \in \Sigma^{*}$. Furthermore, let $L \subseteq \Sigma^{*}$ be a permutation closed language such that the Myhill-Nerode equivalence relation $\sim_{L}$ has only a finite number of positive equivalence classes. Then, the language $w L$ is in ROWJ.

For marked concatenation we find a similar result, which can be deduced from Corollary 4 and Theorem 24, too, because $L_{1} a$ is a prefix-free regular language.

Corollary 26. Let $\Sigma$ be an alphabet and $a \in \Sigma$. Moreover, let $L_{1} \subseteq(\Sigma \backslash\{a\})^{*}$ be a regular language and $L_{2} \subseteq \Sigma^{*}$ be a permutation closed language such that the Myhill-Nerode equivalence relation $\sim_{L_{2}}$ has only a finite number of positive equivalence classes. Then, the language $L_{1} a L_{2}$ is in ROWJ.

Now, we turn back to the closure properties of ROWJ.

Theorem 27. The family ROWJ is not closed under $\lambda$-free homomorphism nor under homomorphism.

Proof. Consider the permutation closed language

$$
L=a^{*} \cup\left\{\left.w \in\{b, c\}^{*}|| w\right|_{b}=|w|_{c}\right\} .
$$

The positive equivalence classes of $\sim_{L}$ are $[\lambda],[a]$, and $[b c]$. So, the language $L$ is in ROWJ, by Corollary 4. Let the $\lambda$-free homomorphism $h:\{a, b, c\}^{*} \rightarrow\{a, b\}^{*}$ be defined by $h(a)=a$, $h(b)=b$, and $h(c)=a$. Then, we get

$$
h(L)=\left\{\left.w \in\{a, b\}^{*}|| w\right|_{b}=0 \vee|w|_{b}=|w|_{a}\right\},
$$

which was shown to be not in ROWJ in Lemma 5.

We also consider the operation of inverse homomorphism:

Theorem 28. The family ROWJ is not closed under inverse homomorphism. 
Proof. Let $A$ be the ROWJFA $A=\left(\left\{q_{0}, q_{1}, q_{2}\right\},\{a, b, c\}, R, q_{0},\left\{q_{0}, q_{2}\right\}\right)$, where $R$ consists of the rules $q_{0} c \rightarrow q_{0}, q_{0} b \rightarrow q_{1}, q_{1} a \rightarrow q_{2}$, and $q_{2} b \rightarrow q_{1}$. The ROWJFA $A$ is depicted in Figure 3 .

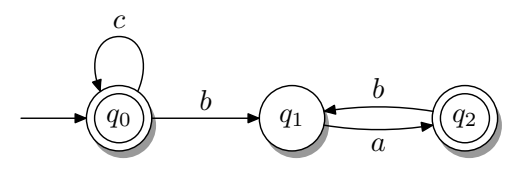

Fig. 3. The ROWJFA $A$ satisfying $L(A) \cap\{a c, b\}^{*}=\left\{(a c)^{n} b^{n} \mid n \geq 0\right\}$.

Let $h:\{a, b\}^{*} \rightarrow\{a, b, c\}^{*}$ be the homomorphism, given by $h(a)=a c$ and $h(b)=b$. It is not hard to see that $h\left(\{a, b\}^{*}\right)=\{a c, b\}^{*}$.

Let now $\lambda \neq w \in L(A) \cap\{a c, b\}^{*}$, which implies $|w|_{b}>0$. When $A$ reads $w$, it reaches the first occurrence of the symbol $b$ in state $q_{0}$. After reading this $b$, the automaton is in state $q_{1}$. Now, no more $c$ can be read. So, we get $w \in(a c)^{+} b^{+}$. Whenever $A$ is in state $q_{2}$, it has read the same number of $a$ 's and $b$ 's. This gives us $w \in\left\{(a c)^{n} b^{n} \mid n>0\right\}$. That shows the invlusion of $L(A) \cap\{a c, b\}^{*}$ within $\left\{(a c)^{n} b^{n} \mid n \geq 0\right\}$

On the other hand, for $n>0$, we have

$$
q_{0}(a c)^{n} b^{n} \circlearrowright^{n} q_{0} b^{n} a^{n} \circlearrowright^{2} q_{2} a^{n-1} b^{n-1} \circlearrowright^{2} q_{2} a^{n-2} b^{n-2} \circlearrowright^{2} \cdots \circlearrowright^{2} q_{2} a b \circlearrowright^{2} q_{2} .
$$

This implies $L(A) \cap\{a c, b\}^{*}=\left\{(a c)^{n} b^{n} \mid n \geq 0\right\}$. We get

$$
\begin{aligned}
h^{-1}(L(A)) & =h^{-1}\left(L(A) \cap h\left(\{a, b\}^{*}\right)\right) \\
& =h^{-1}\left(L(A) \cap\{a c, b\}^{*}\right) \\
& =h^{-1}\left(\left\{(a c)^{n} b^{n} \mid n \geq 0\right\}\right)=\left\{a^{n} b^{n} \mid n \geq 0\right\} .
\end{aligned}
$$

In [3] it was shown that this language is not in ROWJ.

Finally, we take a look at the permutation closure of ROWJ.

Theorem 29. The family ROWJ is not closed under permutation closure.

Proof. By Theorem 11, there is a language $L$, that is in JFA, but not in ROWJ. There exists a DFA $A$ with $L_{J}(A)=L$. Because of $(1)$, we have $\operatorname{perm}\left(L_{R}(A)\right)=L_{J}(A)=L$.

Next, we consider the language family pROWJ in more detail. One can easily find witness languages to see that pROWJ is not closed under union with regular languages, intersection with regular languages, concatenation, concatenation with regular languages (from both sides), Kleene star, Kleene plus, substitution, homomorphism, and $\lambda$-free homomorphism. For all these operations, the witness languages can be chosen in a way such that the resulting language is not even permutation closed. On the other hand, it is not hard to see that the family of permutation closed languages is closed under union, intersection, complement, and inverse homomorphism. We investigate how the language family pROWJ behaves under the latter four operations. From the proofs of the Theorems 21 and 22 we get:

Theorem 30. The family pROWJ is not closed under union and under complement.

The next theorem shows that pROWJ is closed under intersection.

Theorem 31. Let $L_{1} \in \mathbf{p R O W} \mathbf{J}_{\mathbf{m}}$ and $L_{2} \in \mathbf{p R O W}_{\mathbf{n}}$, for some $n, m \geq 0$. Then, the language $L_{1} \cap L_{2} \in \mathbf{p R O W} \mathbf{J}_{\mathbf{m n}}$. 
Proof. Let $\Sigma$ be an alphabet such that $L_{1}, L_{2} \subseteq \Sigma^{*}$. The set $\Sigma^{*}$ is enumerable, so, there is a total order on $\Sigma^{*}$ such that each non-empty subset of $\Sigma^{*}$ has exactly one minimal element. Set

$$
X=\bigcup_{\substack{(S, T) \in\left(L_{1} / \sim_{L_{1}}\right) \times\left(L_{2} / \sim_{L_{2}}\right) \\ \text { with } S \cap T \neq \emptyset}}\{\min (S \cap T)\} .
$$

Because of Theorem 3 we have $\left|L_{1} / \sim_{L_{1}}\right| \leq m$ and $\left|L_{2} / \sim_{L_{2}}\right| \leq n$. That gives us $|X| \leq m n$. Now, let $w \in L_{1} \cap L_{2}$. There exists exactly one

$$
(S, T) \in\left(L_{1} / \sim_{L_{1}}\right) \times\left(L_{2} / \sim_{L_{2}}\right)
$$

such that $w \in S \cap T$. Let $v=\min (S \cap T)$ and $u$ be an arbitrary word in $\Sigma^{*}$. For $i \in\{1,2\}$, we have $w u \in L_{i}$ if and only if $v u \in L_{i}$, because of $w \sim_{L_{i}} v$. This implies that $w u \in L_{1} \cap L_{2}$ if and only if $v u \in L_{1} \cap L_{2}$. We get $w \sim_{L_{1} \cap L_{2}} v$. So, for $\sim_{L_{1} \cap L_{2}}$, we have shown that each element of the intersection $L_{1} \cap L_{2}$ is equivalent to an element out of $X$. It follows that $\sim_{L_{1} \cap L_{2}}$ has at most $m n$ positive equivalence classes. By using Theorem 3 again, we get $L_{1} \cap L_{2} \in \mathbf{p R O W} \mathbf{J}_{\mathbf{m n}}$.

As an immediate consequence we get:

Corollary 32. The family $\mathbf{p R O W J}$ is closed under intersection.

Our next result implies that pROWJ is closed under inverse homomorphism.

Theorem 33. Let $\Gamma$ and $\Sigma$ be alphabets and $h: \Gamma^{*} \rightarrow \Sigma^{*}$ be a homomorphism. Furthermore let $L \subseteq \Sigma^{*}$ be in $\mathbf{p R O W} \mathbf{J}_{\mathbf{n}}$, for some $n \geq 0$. Then, the language $h^{-1}(L)$ is also in $\mathbf{p R O W} \mathbf{J}_{\mathbf{n}}$.

Proof. Theorem 3 gives us $\left|L / \sim_{L}\right| \leq n$. From $L=\bigcup_{S \in L / \sim_{L}} S$, we get

$$
h^{-1}(L)=\bigcup_{S \in L / \sim_{L}} h^{-1}(S) .
$$

Consider now an element $S \in L / \sim_{L}$, two words $v, w \in h^{-1}(S)$, and an arbitrary $u \in \Gamma^{*}$. Because of $h(v), h(w) \in S$, we have $h(v) \sim_{L} h(w)$. It follows that

$$
v u \in h^{-1}(L) \Leftrightarrow h(v) h(u) \in L \Leftrightarrow h(w) h(u) \in L \Leftrightarrow w u \in h^{-1}(L) .
$$

We have shown $v \sim_{h^{-1}(L)} w$. So, we get $\left|h^{-1}(L) / \sim_{h^{-1}(L)}\right| \leq\left|L / \sim_{L}\right| \leq n$, which implies that $h^{-1}(L)$ is in $\mathbf{p R O W} \mathbf{J}_{\mathbf{n}}$, by Theorem 3 .

Thus we immediately get:

Corollary 34. The family $\mathbf{p R O W J}$ is closed under inverse homomorphism.

\section{More on Languages Accepted by ROWJFAs}

In Corollary 4 a characterization of the permutation closed languages that are in ROWJ was given. In this section, we characterize languages in ROWJ for some cases where the considered language does not need to be permutation closed.

Theorem 35. For an alphabet $\Sigma$, let $w \in \Sigma^{*}$ and $L \subseteq \Sigma^{*}$. Then, the language $w L$ is in ROWJ if and only if $L$ is in ROWJ. 
Proof. If $L$ is in ROWJ, then $w L$ is also in ROWJ, because the language family ROWJ is closed under concatenation with prefix-free languages from the left. Now assume that $w L$ is in ROWJ and $L \neq \emptyset$. We may also assume that $|w|=1$. The general case follows from this special case via a trivial induction over the length of $w$. Thus, let $w=a$ for an $a \in \Sigma$ and let $A=(Q, \Sigma, R, s, F)$ be a DFA with $L_{R}(A)=a L$. In the following, we will show via a contradiction that the value $R(s, a)$ is defined. Assume that $R(s, a)$ is undefined and let $v$ be an arbitrary word out of $L$. Because $a v \in L_{R}(A)$, there is a symbol $b \in \Sigma_{s}$, two words $x \in\left(\Sigma \backslash \Sigma_{s}\right)^{*}$ and $y \in \Sigma^{*}$, and a state $p \in F$ such that $v=x b y$ and saxby $R_{(s, b) y a x} \circlearrowright^{*} p$. This gives us sbyax $\vdash R(s, b)$ yax $\circlearrowright^{*} p$, which implies byax $\in L_{R}(A)=a L$. However, this is a contradiction, because $b \neq a$. So, the value $R(s, a)$ is defined.

Consider the DFA $B=(Q, \Sigma, R, R(s, a), F)$. For a word $z \in \Sigma^{*}$, we have $z \in L_{R}(B)$ if and only if $a z \in L_{R}(A)=a L$, because of $s a z \vdash R(s, a) z$. That gives us $L_{R}(B)=L$ and we have shown that $L$ is in ROWJ.

From the previous theorem and Corollary 4 we get a generalization of the latter corollary.

Corollary 36. For an alphabet $\Sigma$, let $w \in \Sigma^{*}$ and let $L \subseteq \Sigma^{*}$ be a permutation closed language. Then, the language $w L$ is in ROWJ if and only if the Myhill-Nerode equivalence relation $\sim_{L}$ has only a finite number of positive equivalence classes.

Next, we will give a characterization for the concatenation $L w$ of a language $L$ and a word $w$. To do so, we need the following lemma. It treats the case of an ROWJFA that is only allowed to jump over one of the input symbols.

Lemma 37. Let $A=(Q, \Sigma, R, s, F)$ be a DFA with a symbol $a \in \Sigma$ such that the value $R(q, b)$ is defined for all $(q, b) \in Q \times(\Sigma \backslash\{a\})$. Then, the language $L_{R}(A)$ is regular.

Proof. Consider the DFA

$$
B=\left(Q \times(Q \cup\{d\})^{Q}, \Sigma, S,\left(s, \operatorname{id}_{Q \rightarrow Q \cup\{d\}}\right), G\right),
$$

where $d \notin Q \cup \Sigma$, the map $\operatorname{id}_{Q \rightarrow Q \cup\{d\}}$ is the identity map and

$$
G=\left\{(q, f) \in Q \times(Q \cup\{d\})^{Q} \mid f(q) \in F\right\} .
$$

The total map $S$ is defined as follows:

$$
S((q, f), b)= \begin{cases}(R(q, b), f) & \text { if }(q, f) \in Q \times(Q \cup\{d\})^{Q} \text { and } b \in \Sigma_{R, q} \\ (q, g \circ f) & \text { if }(q, f) \in Q \times(Q \cup\{d\})^{Q} \text { and } b \notin \Sigma_{R, q} .\end{cases}
$$

The map $g:(Q \cup\{d\}) \rightarrow(Q \cup\{d\})$ is defined in the following way: let

$$
g(p)= \begin{cases}R(p, a) & \text { if } p \in Q \text { and } a \in \Sigma_{R, p} \\ d & \text { if } p \in Q \text { and } a \notin \Sigma_{R, p} \\ d & \text { otherwise. }\end{cases}
$$

This completes the description of $B$. We will show that $L_{D}(B)=L_{R}(A)$.

Let $w \in \Sigma^{*}$. We decompose the word $w$ into factors that are consumed by $A$ and factors that are jumped over by the automaton in question: there exists a number $m>0$, 
words $w_{1}, w_{2}, \ldots, w_{m} \in \Sigma^{*}$, symbols $b_{2}, b_{3}, \ldots, b_{m} \in \Sigma \backslash\{a\}$, numbers $n_{1}, \ldots, n_{m} \in \mathbb{N}$ such that $n_{1} n_{2} \cdots n_{m-1}>0$, and states $p_{1}, p_{2}, \ldots, p_{m} \in Q$ and $q_{2}, q_{3}, \ldots, q_{m} \in Q$ with

$$
\begin{aligned}
s w=s w_{1} a^{n_{1}} \prod_{i=2}^{m} b_{i} w_{i} a^{n_{i}} & \vdash_{A}^{\left|w_{1}\right|} p_{1} a^{n_{1}} \prod_{i=2}^{m} b_{i} w_{i} a^{n_{i}} \\
& \circlearrowright_{A} q_{2} w_{2} a^{n_{2}}\left(\prod_{i=3}^{m} b_{i} w_{i} a^{n_{i}}\right) a^{n_{1}} \\
& \vdash_{A}^{\left|w_{2}\right|} p_{2} a^{n_{2}}\left(\prod_{i=3}^{m} b_{i} w_{i} a^{n_{i}}\right) a^{n_{1}} \\
& \circlearrowright_{A} q_{3} w_{3} a^{n_{3}}\left(\prod_{i=4}^{m} b_{i} w_{i} a^{n_{i}}\right) a^{n_{1}+n_{2}} \\
& \ldots \\
& \vdash_{A}^{\left|w_{m-1}\right|} p_{m-1} a^{n_{m-1}} b_{m} w_{m} a^{n_{m}+\sum_{i=1}^{m-2} n_{i}} \\
& \circlearrowright_{A} q_{m} w_{m} a^{\sum_{i=1}^{m} n_{i}} \\
& \vdash_{A}^{\left|w_{m}\right|} p_{m} a^{\sum_{i=1}^{m} n_{i}} .
\end{aligned}
$$

We have $w \in L_{R}(A)$ if and only if $g^{\sum_{i=1}^{m} n_{i}}\left(p_{m}\right) \in F$. On the other hand, we get the following computation

$$
\begin{aligned}
\left(s, \operatorname{id}_{Q \rightarrow Q \cup\{d\}}\right) w & =\left(s, \operatorname{id}_{Q \rightarrow Q \cup\{d\}}\right) w_{1} a^{n_{1}} \prod_{i=2}^{m} b_{i} w_{i} a^{n_{i}} \\
& \vdash_{B}^{\left|w_{1}\right|}\left(p_{1}, \operatorname{id}_{Q \rightarrow Q \cup\{d\}}\right) a^{n_{1}} \prod_{i=2}^{m} b_{i} w_{i} a^{n_{i}} \\
& \vdash_{B}^{n_{1}+1}\left(q_{2},\left.g^{n_{1}}\right|_{Q}\right) w_{2} a^{n_{2}}\left(\prod_{i=3}^{m} b_{i} w_{i} a^{n_{i}}\right) \\
& \vdash_{B}^{\left|w_{2}\right|}\left(p_{2},\left.g^{n_{1}}\right|_{Q}\right) a^{n_{2}}\left(\prod_{i=3}^{m} b_{i} w_{i} a^{n_{i}}\right) \\
& \vdash_{B}^{n_{2}+1}\left(q_{3},\left.g^{n_{1}+n_{2}}\right|_{Q}\right) w_{3} a^{n_{3}}\left(\prod_{i=4}^{m} b_{i} w_{i} a^{n_{i}}\right) \\
& \ldots \\
& \vdash_{B}^{\left|w_{m-1}\right|}\left(p_{m-1},\left.g^{\sum_{i=1}^{m-2} n_{i}}\right|_{Q}\right) a^{n_{m-1}} b_{m} w_{m} a^{n_{m}} \\
& \vdash_{B}^{n_{m-1}+1}\left(q_{m},\left.g^{\sum_{i=1}^{m-1} n_{i}}\right|_{Q}\right) w_{m} a^{n_{m}} \\
& \vdash_{B}^{\left|w_{m}\right|}\left(p_{m},\left.g^{\sum_{i=1}^{m-1} n_{i}}\right|_{Q}\right) a^{n_{m}} .
\end{aligned}
$$

Set $k=\max \left\{r \in\left\{0,1, \ldots, n_{m}\right\} \mid g^{r}\left(p_{m}\right) \in Q\right\}$. That gives

$$
\begin{aligned}
\left(p_{m},\left.g^{\sum_{i=1}^{m-1} n_{i}}\right|_{Q}\right) a^{n_{m}} & \vdash_{B}^{k}\left(g^{k}\left(p_{m}\right),\left.g^{\sum_{i=1}^{m-1} n_{i}}\right|_{Q}\right) a^{n_{m}-k} \\
& \vdash_{B}^{n_{m}-k}\left(g^{k}\left(p_{m}\right),\left.g^{\sum_{i=1}^{m} n_{i}-k}\right|_{Q}\right) .
\end{aligned}
$$

Thus, we have $w \in L_{D}(B)$ if and only if

$$
g^{\sum_{i=1}^{m} n_{i}}\left(p_{m}\right)=g^{\sum_{i=1}^{m} n_{i}-k}\left(g^{k}\left(p_{m}\right)\right) \in F,
$$


which holds if and only if $w \in L_{R}(A)$. That shows $L_{D}(B)=L_{R}(A)$ and that $L_{R}(A)$ is a regular language. This proves the lemma.

Our characterization for languages of the form $L w$ generalizes a result from [3], which says that the language $\left\{\left.v a\left|v \in\{a, b\}^{*},\right| v\right|_{a}=|v|_{b}\right\}$ is not in ROWJ:

Theorem 38. For an alphabet $\Sigma$, let $w \in \Sigma^{*}$ be a non-empty word and $L \subseteq \Sigma^{*}$. Then, the language $L w$ is in ROWJ if and only if $L$ is regular.

Proof. If $L$ is regular, then $L w$ is also regular, which means that $L w$ is in ROWJ. Assume now, that $L w$ is in ROWJ. As in the proof of Theorem 35, we can assume that $w=a$ for an $a \in \Sigma$. Let $A=(Q, \Sigma, R, s, F)$ be a DFA with $L_{R}(A)=L a$. Consider the DFA $B=(Q \cup\{d\}, \Sigma, S, s, F)$ and let $d$ be a new symbol with $d \notin Q \cup \Sigma$. The map $S$ is defined as follows: for $(q, b) \in Q \times \Sigma$, we set $S(q, b)=R(q, b)$, if $R(q, b)$ is defined. If $R(q, b)$ is undefined and $b \neq a$, we define $S(q, b)=d$. For all $q \in Q$, the value $S(q, a)$ is undefined, if $R(q, a)$ is undefined. Finally, for all $b \in \Sigma$, it holds $S(d, b)=d$. By Lemma 37, the language $L_{R}(B)$ is regular. We will show that

$$
L_{R}(A)=L_{R}(B)
$$

Then, the regularity of $L a=L_{R}(A)$ implies the regularity of $L$, because regular languages are closed under the operation of quotient with a regular language.

First, let $v \in L_{R}(B)$ and $f \in F$ with $s v \circlearrowright_{B}^{*} f$. For a state $q \in Q$, a symbol $b \in \Sigma_{S, q}$, and words $x \in\left(\Sigma \backslash \Sigma_{S, q}\right)^{*}$ and $y \in \Sigma^{*}$ with $s v \circlearrowright_{B}^{*} q x b y$, we have $x \in\left(\Sigma \backslash \Sigma_{R, q}\right)^{*}$ and

$$
q x b y \circlearrowright_{B} S(q, b) y x \circlearrowright_{B}^{*} f .
$$

This implies $S(q, b) \neq d$, which tells us $b \in \Sigma_{R, q}$ and $R(q, b)=S(q, b)$. We get $q x b y \circlearrowright_{A} S(q, b) y x$. By induction, we see that $s v \circlearrowright_{A}^{*} f$. Therefore, we have $v \in L_{R}(A)$.

Now, let $v \in L_{R}(A)$ and $f \in F$ with $s v \circlearrowright_{A}^{*} f$. Assume that $v \notin L_{R}(B)$. Then, there exists a symbol out of $\Sigma \backslash\{a\}$ that is jumped over during the processing of $A$, when the starting configuration is $s v$. The part of $v$ that is visited by $A$ before it jumps over the first symbol out of $\Sigma \backslash\{a\}$ will be decomposed into factors that are consumed by $A$ and factors that are jumped over by the device under consideration: there is a natural number $m>0$, words $w_{1}, w_{2}, \ldots, w_{m+2} \in \Sigma^{*}$, symbols $b_{2}, b_{3}, \ldots, b_{m+1} \in \Sigma \backslash\{a\}$ and $c \in \Sigma$, numbers $n_{1}, \ldots, n_{m} \in \mathbb{N}$ with $n_{1} n_{2} \cdots n_{m-1}>0$, and states $p_{1}, p_{2}, \ldots, p_{m}, q_{2}, q_{3}, \ldots, q_{m+1} \in Q$ with symbols $b_{i+1} \in \Sigma_{p_{i}}$, for every $i$ satisfying $i \in\{1,2, \ldots, m-1\}$, such that word $a^{n_{m}} b_{m+1} w_{m+1} \in\left(\Sigma \backslash \Sigma_{R, p_{m}}\right)^{+}, c \in \Sigma_{R, p_{m}}$, and

$$
\begin{aligned}
& s v=s w_{1} a^{n_{1}}\left(\prod_{i=2}^{m} b_{i} w_{i} a^{n_{i}}\right) b_{m+1} w_{m+1} c w_{m+2} \\
& \vdash_{A}^{\left|w_{1}\right|} p_{1} a^{n_{1}}\left(\prod_{i=2}^{m} b_{i} w_{i} a^{n_{i}}\right) b_{m+1} w_{m+1} c w_{m+2} \\
& \circlearrowright_{A} q_{2} w_{2} a^{n_{2}}\left(\prod_{i=3}^{m} b_{i} w_{i} a^{n_{i}}\right) b_{m+1} w_{m+1} c w_{m+2} a^{n_{1}}
\end{aligned}
$$


continued by

$$
\begin{aligned}
q_{2} w_{2} a^{n_{2}}\left(\prod_{i=3}^{m} b_{i} w_{i} a^{n_{i}}\right) & b_{m+1} w_{m+1} c w_{m+2} a^{n_{1}} \\
& \vdash_{A}^{\left|w_{2}\right|} p_{2} a^{n_{2}}\left(\prod_{i=3}^{m} b_{i} w_{i} a^{n_{i}}\right) b_{m+1} w_{m+1} c w_{m+2} a^{n_{1}} \\
& \circlearrowright_{A} q_{3} w_{3} a^{n_{3}}\left(\prod_{i=4}^{m} b_{i} w_{i} a^{n_{i}}\right) b_{m+1} w_{m+1} c w_{m+2} a^{n_{1}+n_{2}} \\
& \ldots \\
& \vdash_{A}^{\left|w_{m-1}\right|} p_{m-1} a^{n_{m-1}} b_{m} w_{m} a^{n_{m}} b_{m+1} w_{m+1} c w_{m+2} a^{\sum_{i=1}^{m-2} n_{i}} \\
& \circlearrowright_{A} q_{m} w_{m} a^{n_{m}} b_{m+1} w_{m+1} c w_{m+2} a^{\sum_{i=1}^{m-1} n_{i}} \\
& \vdash_{A}^{\left|w_{m}\right|} p_{m} a^{n_{m}} b_{m+1} w_{m+1} c w_{m+2} a^{\sum_{i=1}^{m-1} n_{i}} \\
& \circlearrowright_{A} q_{m+1} w_{m+2} a^{\sum_{i=1}^{m} n_{i}} b_{m+1} w_{m+1} \circlearrowright_{A}^{*} f .
\end{aligned}
$$

We get

$$
\begin{aligned}
& s w_{1}\left(\prod_{i=2}^{m} b_{i} w_{i}\right) w_{m+1} c w_{m+2} a^{\sum_{i=1}^{m} n_{i}} b_{m+1} \\
& \vdash_{A}^{\left|w_{1} \prod_{i=2}^{m} b_{i} w_{i}\right|} p_{m} w_{m+1} c w_{m+2} a^{\sum_{i=1}^{m} n_{i}} b_{m+1} \\
& \circlearrowright_{A} q_{m+1} w_{m+2} a^{\sum_{i=1}^{m} n_{i}} b_{m+1} w_{m+1} \circlearrowright_{A}^{*} f .
\end{aligned}
$$

This implies

$$
w_{1}\left(\prod_{i=2}^{m} b_{i} w_{i}\right) w_{m+1} c w_{m+2} a^{\sum_{i=1}^{m} n_{i}} b_{m+1} \in L_{R}(A)=L a,
$$

a contradiction. This shows $v \in L_{R}(B)$, which proves the theorem.

Now, we consider the case of two languages over disjoint alphabets.

Theorem 39. For disjoint alphabets $\Sigma_{1}$ and $\Sigma_{2}$, let languages $L_{1} \subseteq \Sigma_{1}^{*}$ and $L_{2} \subseteq \Sigma_{2}^{*}$ satisfy $L_{1} \neq \emptyset \neq L_{2} \neq\{\lambda\}$ such that $L_{1} L_{2}$ is in ROWJ. Then, the language $L_{1}$ is regular and $L_{2}$ is in ROWJ.

Proof. The proof is similar as those of Theorem 35. Let $A=\left(Q, \Sigma=\Sigma_{1} \cup \Sigma_{2}, R, s, F\right)$ be a DFA with $L_{R}(A)=L_{1} L_{2}$. For an $m \geq 0$ and $a_{1}, a_{2}, \ldots, a_{m} \in \Sigma_{1}$, let $w=a_{1} a_{2} \ldots a_{m} \in L_{1}$. We will show by induction that for each $0 \leq n \leq m$, there is a state $q_{n} \in Q$ with $s w \vdash^{n} q_{n} a_{n+1} a_{n+2} \ldots a_{m}$. For $n=0$, we just set $q_{0}=s$. Assume that, for a fixed $k$ with $0 \leq k<m$, we already know that there is a state $q_{k} \in Q$ with

$$
s w \vdash^{k} q_{k} a_{k+1} a_{k+2} \ldots a_{m} .
$$

If the value $R\left(q_{k}, a_{k+1}\right)$ is defined, then we have

$$
s w \vdash^{k+1} R\left(q_{k}, a_{k+1}\right) a_{k+2} a_{k+3} \ldots a_{m} .
$$

Therefore, now let $R\left(q_{k}, a_{k+1}\right)$ be undefined and let $v$ be an arbitrary non-empty word out of $L_{2}$. We get

$$
s w v \vdash^{k} q_{k} a_{k+1} a_{k+2} \ldots a_{m} v .
$$


Because of $w v \in L_{R}(A)$, there exist a symbol $b \in \Sigma_{q_{k}}$, words $x \in\left(\Sigma \backslash \Sigma_{q_{k}}\right)^{*}$ and $y \in \Sigma^{*}$, and a state $p \in F$ such that $a_{k+2} a_{k+3} \ldots a_{m} v=x b y$ and

$$
q_{k} a_{k+1} x b y \circlearrowright R\left(q_{k}, b\right) y a_{k+1} x \circlearrowright^{*} p .
$$

This implies

$$
s a_{1} a_{2} \ldots a_{k} x b y a_{k+1} \vdash^{k} q_{k} x b y a_{k+1} \circlearrowright R\left(q_{k}, b\right) y a_{k+1} x \circlearrowright^{*} p,
$$

which gives us that $a_{1} a_{2} \ldots a_{k} x b y a_{k+1} \in L_{R}(A)$. However, this word is equal to

$$
a_{1} a_{2} \ldots a_{k} a_{k+2} a_{k+3} \ldots a_{m} v a_{k+1}
$$

and we have

$$
a_{1} a_{2} \ldots a_{k} a_{k+2} a_{k+3} \ldots a_{m} v a_{k+1} \in \Sigma_{1}^{*} \Sigma_{2}^{+} \Sigma_{1} \subseteq \Sigma^{*} \backslash\left(\Sigma_{1}^{*} \Sigma_{2}^{*}\right) \subseteq \Sigma^{*} \backslash\left(L_{1} L_{2}\right),
$$

which is a contradiction. So, the value $R\left(q_{k}, a_{k+1}\right)$ has to be defined and we have shown by induction that for each $0 \leq n \leq m$, there is a state $q_{n} \in Q$ with $s w \vdash^{n} q_{n} a_{n+1} a_{n+2} \ldots a_{m}$. We set $q_{w}=q_{m}$ and get $s w \vdash|w| q_{w}$.

For every $w \in L_{1}$, we consider the DFA $B_{w}=\left(Q, \Sigma_{2},\left.R\right|_{Q \times \Sigma_{2}}, q_{w}, F\right)$. For every $v \in \Sigma_{2}^{*}$, we have

$$
\begin{aligned}
v \in L_{R}\left(B_{w}\right) & \Leftrightarrow\left(\exists f \in F: q_{w} v \circlearrowright_{B_{w}}^{*} f\right) \\
& \Leftrightarrow\left(\exists f \in F: s w v \circlearrowright_{A}^{*} f\right) \\
& \Leftrightarrow w v \in L_{R}(A)=L_{1} L_{2} \\
& \Leftrightarrow v \in L_{2} .
\end{aligned}
$$

This shows $L_{R}\left(B_{w}\right)=L_{2}$, so $L_{2}$ is in ROWJ.

For every $v \in L_{2}$, we define the set $Q_{v}=\left\{q \in Q \mid \exists f \in F: q v \circlearrowright_{A}^{*} f\right\}$ and the determinstic finite stace device $C_{v}=\left(Q, \Sigma_{1},\left.R\right|_{Q \times \Sigma_{1}}, s, Q_{v}\right)$. For every $w \in \Sigma_{1}^{*}$, we have

$$
\begin{aligned}
w \in L_{D}\left(C_{v}\right) & \Leftrightarrow\left(\exists q \in Q_{v}: s w \vdash_{C_{v}}^{*} q\right) \\
& \Leftrightarrow\left(\exists q \in Q, f \in F: s w v \vdash_{A}^{*} q v \circlearrowright_{A}^{*} f\right) \\
& \Leftrightarrow w v \in L_{R}(A)=L_{1} L_{2} \\
& \Leftrightarrow w \in L_{1} .
\end{aligned}
$$

Therefore, we conclude $L_{D}\left(C_{v}\right)=L_{1}$, so $L_{1}$ is regular, which proves the theorem.

Adding prefix-freeness for $L_{1}$, we get an equivalence, by Theorem 39 and the closure of ROWJ under left-concatenation with prefix-free regular sets.

Corollary 40. For disjoint alphabets $\Sigma_{1}$ and $\Sigma_{2}$, let $L_{1} \subseteq \Sigma_{1}^{*}$ be a prefix-free set and $L_{2} \subseteq \Sigma_{2}^{*}$ be an arbitrary language with $L_{1} \neq \emptyset \neq L_{2} \neq\{\lambda\}$. Then, the language $L_{1} L_{2}$ is in ROWJ if and only if $L_{1}$ is regular and $L_{2}$ is in $\mathbf{R O W J}$.

The previous corollary directly implies the following characterization that is another generalization of Corollary 4.

Corollary 41. For disjoint alphabets $\Sigma_{1}$ and $\Sigma_{2}$, let $L_{1} \subseteq \Sigma_{1}^{*}$ be a prefix-free set and $L_{2} \subseteq \Sigma_{2}^{*}$ be a permutation closed language with $L_{1} \neq \emptyset \neq L_{2} \neq\{\lambda\}$. Then, the language $L_{1} L_{2}$ is in ROWJ if and only if $L_{1}$ is regular and the Myhill-Nerode equivalence relation $\sim_{L_{2}}$ has only a finite number of positive equivalence classes. 
If a non-empty language and a non-empty permutation closed language over disjoint alphabets are separated by a symbol, we get the following result:

Corollary 42. For disjoint alphabets $\Sigma_{1}$ and $\Sigma_{2}$, let $L_{1} \subseteq \Sigma_{1}^{*}$ be non empty and $L_{2} \subseteq \Sigma_{2}^{*}$ be a non-empty permutation closed language. Furthermore, let $a \in \Sigma_{2}$. Then, the language $L_{1} a L_{2}$ is in ROWJ if and only if $L_{1}$ is regular and the Myhill-Nerode equivalence relation $\sim_{L_{2}}$ has only a finite number of positive equivalence classes.

Proof. If $L_{1}$ is regular and the Myhill-Nerode equivalence relation $\sim_{L_{2}}$ has only a finite number of positive equivalence classes, Corollary 26 tells us that $L_{1} a L_{2}$ is in ROWJ.

Now, assume that $L_{1} a L_{2}$ is in ROWJ. From Theorem 39 we get that $L_{1}$ is regular and $a L_{2}$ is in ROWJ. Corollary 36 gives us that $\sim_{L_{2}}$ has only a finite number of positive equivalence classes.

For an alphabet $\Sigma=\left\{a_{1}, a_{2}, \ldots, a_{n}\right\}$, the family of subsets of $a_{1}^{*} a_{2}^{*} \ldots a_{n}^{*}$ is kind of a counterpart of the family of permutation closed languages over $\Sigma$. In a permutation closed language $L$, for each word $w \in L$, all permutations of $w$ are also in $L$. In a language $M \subseteq a_{1}^{*} a_{2}^{*} \ldots a_{n}^{*}$, for each word $w \in M$, no other permutation of $w$ is in $M$. We can characterize the subsets of $a_{1}^{*} a_{2}^{*} \ldots a_{n}^{*}$ that are in ROWJ. The following lemma helps us to do so.

Lemma 43. Let $A$ be a DFA with input alphabet $\left\{a_{1}, a_{2}, \ldots, a_{n}\right\}$ accepting a letter bounded language, i.e., $L_{R}(A) \subseteq a_{1}^{*} a_{2}^{*} \ldots a_{n}^{*}$. Then, $L_{R}(A)=L_{D}(A)$.

Proof. Because of $(1)$ we have $L_{D}(A) \subseteq L_{R}(A)$. Now assume that $w \in L_{R}(A)$. Again, because of this inclusion chain, there is a permutation $v$ ofthe word $w$ with $v \in L_{D}(A) \subseteq L_{R}(A)$. Since $L_{R}(A) \subseteq a_{1}^{*} a_{2}^{*} \ldots a_{n}^{*}$, we conclude that $w=v \in L_{D}(A)$. Thus, $L_{R}(A)=L_{D}(A)$.

In [3] it was shown that the language $\left\{a^{n} b^{n} \mid n \geq 0\right\}$ is not in ROWJ. Our characterization generalizes this result:

Theorem 44. Let $\left\{a_{1}, a_{2}, \ldots, a_{n}\right\}$ be an alphabet and $L \subseteq a_{1}^{*} a_{2}^{*} \ldots a_{n}^{*}$. Then, the language $L$ is in ROWJ if and only if $L$ is regular.

Proof. If $L$ is regular, then $L$ is also in ROWJ, because of REG $\subset$ ROWJ. If $L$ is in ROWJ, then there exists a DFA $A$ with $L=L_{R}(A)$. Because of Lemma 43 , we get $L=L_{R}(A)=L_{D}(A)$, which is a regular language. This proves the stated claim.

\section{References}

1. S. Beier, M. Holzer, and M. Kutrib. Operational state complexity and decidability of jumping finite automata. In É. Charlier, J. Leroy, and M. Rigo, editors, Proceedings of the 21 st International Conference on Developments in Language Theory, number 10396 in LNCS, pages 96-108, Liège, Belgium, 2017. Springer.

2. S. Bensch, H. Bordihn, M. Holzer, and M. Kutrib. On input-revolving deterministic and nondeterministic finite automata. Inform. Comput., 207(11):1140-1155, 2009.

3. H. Chigahara, S. Fazekas, and A. Yamamura. One-way jumping finite automata. Internat. J. Found. Comput. Sci., 27(3):391-405, 2016.

4. C. Culy. Formal properties of natural language and linguistic theories. Linguistics and Philosophy, 19:599-617, 1996.

5. L. E. Dickson. Finiteness of the odd perfect and primitive abundant numbers with $n$ distinct prime factors. American J. Math., 35(4):413-422, 1913.

6. H. Fernau, M. Paramasivan, and M. L. Schmid. Jumping finite automata: Characterizations and compliexity. In F. Drewes, editor, Proceedings of the 20th Conference on Implementation and Application of Automata, number 9223 in LNCS, pages 89-101, Umeå, Sweden, 2015. Springer. 
7. H. Fernau, M. Paramasivan, M. L. Schmid, and V. Vorel. Characterization and complexity results on jumping finite automata. http://arxiv.org/abs/1512.00482, 2015.

8. S. Ginsburg and E. H. Spanier. Bounded ALGOL-like languages. Trans. AMS, 113:333-368, 1964.

9. S. A. Greibach. An infinite hierarchy of context-free languages. J. ACM, 16(1):91-106, 1969.

10. J. E. Hopcroft and J. D. Ullman. Introduction to Automata Theory, Languages and Computation. AddisonWesley, 1979.

11. A. Meduna and P. Zemek. Jumping finite automata. Internat. J. Found. Comput. Sci., 23(7):1555-1578, 2012 .

12. A. Meduna and P. Zemek. Regulated Grammars and Automata, chapter 17: Jumping Finite Automata, pages 567-585. Springer, 2014.

13. R. J. Parikh. On context-free languages. J. ACM, 13(4):570-581, 1966

14. V. Vorel. Basic properties of jumping finite automata. http://arxiv.org/abs/1511.08396v2, 2015. 


\section{Institut für Informatik}

Justus-Liebig-Universität Giessen

Arndtstr. 2, 35392 Giessen, Germany

\section{Recent Reports}

(Further reports are available at www.informatik.uni-giessen.de.)

B. Truthe, Hierarchy of Subregular Language Families, Report 1801, February 2018.

M. Holzer, M. Hospodár, On the Magic Number Problem of the Cut Operation, Report 1703, October 2017.

M. Holzer, S. Jakobi, A Note on the Computational Complexity of Some Problems for Self-Verifying Finite Automata, Report 1702, April 2017.

S. Beier, M. Holzer, M. Kutrib, On the Descriptional Complexity of Operations on Semilinear Sets, Report 1701, April 2017.

M. Holzer, S. Jakobi, M. Wendlandt, On the Computational Complexity of Partial Word Automata Problems, Report 1404, May 2014.

H. Gruber, M. Holzer, Regular Expressions From Deterministic Finite Automata, Revisited, Report 1403, May 2014.

M. Kutrib, A. Malcher, M. Wendlandt, Deterministic Set Automata, Report 1402, April 2014.

M. Holzer, S. Jakobi, Minimal and Hyper-Minimal Biautomata, Report 1401, March 2014.

J. Kari, M. Kutrib, A. Malcher (Eds.), 19th International Workshop on Cellular Automata and Discrete Complex Systems AUTOMATA 2013 Exploratory Papers, Report 1302, September 2013.

M. Holzer, S. Jakobi, Minimization, Characterizations, and Nondeterminism for Biautomata, Report 1301, April 2013.

A. Malcher, K. Meckel, C. Mereghetti, B. Palano, Descriptional Complexity of Pushdown Store Languages, Report 1203, May 2012.

M. Holzer, S. Jakobi, On the Complexity of Rolling Block and Alice Mazes, Report 1202, March 2012.

M. Holzer, S. Jakobi, Grid Graphs with Diagonal Edges and the Complexity of Xmas Mazes, Report 1201, January 2012.

H. Gruber, S. Gulan, Simplifying Regular Expressions: A Quantitative Perspective, Report 0904, August 2009.

M. Kutrib, A. Malcher, Cellular Automata with Sparse Communication, Report 0903, May 2009.

M. Holzer, A. Maletti, An $n \log n$ Algorithm for Hyper-Minimizing States in a (Minimized) Deterministic Automaton, Report 0902, April 2009.

H. Gruber, M. Holzer, Tight Bounds on the Descriptional Complexity of Regular Expressions, Report 0901, February 2009.

M. Holzer, M. Kutrib, and A. Malcher (Eds.), 18. Theorietag Automaten und Formale Sprachen, Report 0801, September 2008.

M. Holzer, M. Kutrib, Flip-Pushdown Automata: Nondeterminism is Better than Determinism, Report 0301, February 2003

M. Holzer, M. Kutrib, Flip-Pushdown Automata: $k+1$ Pushdown Reversals are Better Than $k$, Report 0206, November 2002

M. Holzer, M. Kutrib, Nondeterministic Descriptional Complexity of Regular Languages, Report 0205, September 2002

H. Bordihn, M. Holzer, M. Kutrib, Economy of Description for Basic Constructions on Rational Transductions, Report 0204, July 2002

M. Kutrib, J.-T. Löwe, String Transformation for $n$-dimensional Image Compression, Report 0203 , May 2002

A. Klein, M. Kutrib, Grammars with Scattered Nonterminals, Report 0202, February 2002

A. Klein, M. Kutrib, Self-Assembling Finite Automata, Report 0201, January 2002

M. Holzer, M. Kutrib, Unary Language Operations and its Nondeterministic State Complexity, Report 0107, November 2001

A. Klein, M. Kutrib, Fast One-Way Cellular Automata, Report 0106, September 2001

M. Holzer, M. Kutrib, Improving Raster Image Run-Length Encoding Using Data Order, Report 0105, July 2001

M. Kutrib, Refining Nondeterminism Below Linear-Time, Report 0104, June 2001

M. Holzer, M. Kutrib, State Complexity of Basic Operations on Nondeterministic Finite Automata, Report 0103, April 2001 